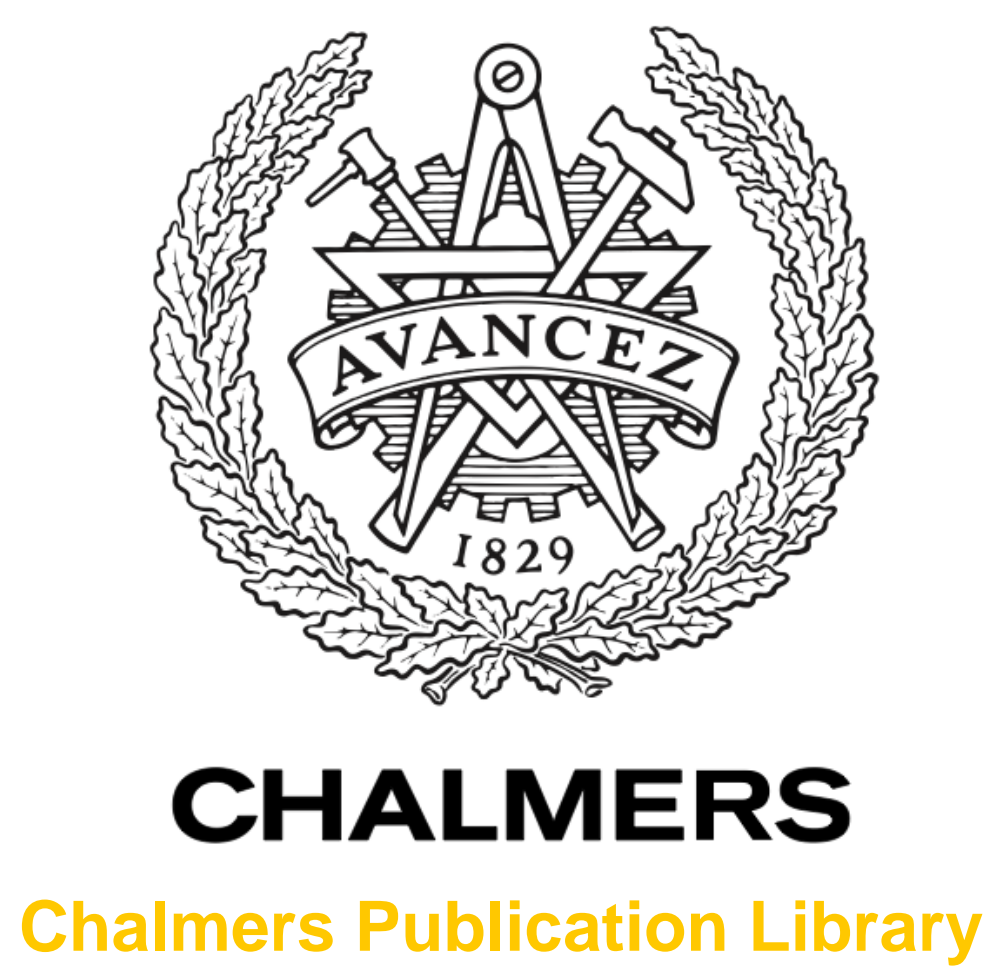

Nonlinear impairment aware resource allocation in elastic optical networks

This document has been downloaded from Chalmers Publication Library (CPL). It is the author's version of a work that was accepted for publication in:

2015 Optical Fiber Communications Conference and Exhibition, OFC 2015; Los Angeles; United States; 22 March 2015 through 26 March 2015

Citation for the published paper:

Zhao, J. ; Wymeersch, H. ; Agrell, E. (2015) "Nonlinear impairment aware resource allocation in elastic optical networks". 2015 Optical Fiber Communications Conference and Exhibition, OFC 2015; Los Angeles; United States; 22 March 2015 through 26 March 2015, vol. June 2015(7121502),

Downloaded from: http://publications.lib.chalmers.se/publication/225898

Notice: Changes introduced as a result of publishing processes such as copy-editing and formatting may not be reflected in this document. For a definitive version of this work, please refer to the published source. Please note that access to the published version might require a subscription.

Chalmers Publication Library (CPL) offers the possibility of retrieving research publications produced at Chalmers University of Technology. It covers all types of publications: articles, dissertations, licentiate theses, masters theses, conference papers, reports etc. Since 2006 it is the official tool for Chalmers official publication statistics. To ensure that Chalmers research results are disseminated as widely as possible, an Open Access Policy has been adopted.

The CPL service is administrated and maintained by Chalmers Library. 


\title{
Nonlinear Impairment Aware Resource Allocation in Elastic Optical Networks
}

\author{
Juzi Zhao, Henk Wymeersch, and Erik Agrell \\ Department of Signals and Systems, Chalmers University of Technology, Gothenburg, Sweden \\ juzi@chalmers.se
}

\begin{abstract}
We propose a novel integer linear program formulation and low-complexity heuristics for nonlinear impairment-aware routing and spectrum allocation in OOFDM-based elastic optical networks. Results indicate $23 \%$ bandwidth reduction compared with a transmission reach-based benchmark method.
\end{abstract}

OCIS codes: 060.4251 Networks, assignment and routing algorithms, 060.4256 Networks, network optimization.

\section{Introduction}

Elastic optical networks based on optical orthogonal frequency division multiplexing (OOFDM) are promising candidates for carrying heterogeneous traffic in next generation networks due to their ability to efficiently allocate fiber bandwidth. In particular, OOFDM subcarriers have much finer bandwidth granularity than wavelengths in WDMbased optical network. A variety of modulation schemes can be used to modulate the OODFM subcarriers and result in different bit rates; therefore, just the required number of subcarriers will be allocated to a service [1]. As in wavelength-division multiplexed (WDM) optical networks, physical impairments exist in OOFDM networks. Most existing related work on impairment-aware routing and wavelength assignment (RWA) in elastic optical networks are based on transmission-reach limits [2,5], where each modulation format has a reach limit. In addition, a guardband consisting of an integer number of subcarriers is placed between two adjacent subcarrier bands assigned to different connections to avoid interference. However, this kind of method fails to consider the dynamic network/connection states. Hence, the reach and guardband may overestimate or underestimate the connection impairments. More sophisticated methods to account for nonlinear interference were studied in [6] for RWA in WDM networks, in [7] for RWA in elastic networks for dynamic traffic, and in [8] for sequentially loaded traffic.

In this paper, we address the problem of routing and spectrum allocation for offline (given) connection requests in transparent elastic optical networks with OOFDM. An analytical model is utilized to calculate the nonlinear physical impairments of each connection.

\section{Model and Problem Statement}

We adopt the physical layer model in [9]. The signal-to-noise ratio (SNR) for connection $i$ using route $r_{i}$ is $\mathrm{SNR}_{i}=$ $G /\left(G_{\mathrm{ASE}}+G_{\mathrm{NLI}}\right)$, where $G$ is the signal power spectral density (PSD) and $G_{\mathrm{ASE}}$ and $G_{\mathrm{NLI}}$ will be defined next. The PSD of the amplified spontaneous emission (ASE) noise is $G_{\mathrm{ASE}}=\sum_{l \in r_{i}} N_{l} G_{\mathrm{ASE}}^{0}$, where $G_{\mathrm{ASE}}^{0}=\left(e^{\alpha L}-1\right) F h v ; N_{l}$ is the number of spans on link $l ; L$ is the length of each span; $\alpha$ is the power attenuation; $F$ is the spontaneous emission factor; $h$ is Planck's constant; and $v$ is the light frequency. The PSD of the noise from nonlinear impairments (NLI) is $G_{\mathrm{NLI}}=\sum_{l \in r_{i}} G_{\mathrm{NLI}}^{l} N_{l}$, where the PSD of the NLI noise in a single span on link $l$ is $G_{\mathrm{NLI}}^{l}=\mu\left(\operatorname{asinh}\left(\rho B_{i}^{2}\right)+\sum_{j} \ln \left[\left(\Delta f_{i j}+\right.\right.\right.$ $\left.\left.\left.B_{j} / 2\right) /\left(\Delta f_{i j}-B_{j} / 2\right)\right]\right)$. In this expression, $\mu=\left(3 \gamma^{2} G^{3}\right) /\left(2 \pi \alpha\left|\beta_{2}\right|\right) ; \rho=\left(\pi^{2}\left|\beta_{2}\right|\right) /(2 \alpha) ; j$ is another connection using link $l ; B_{i}$ and $B_{j}$ are the bandwidths for connections $i$ and $j$, respectively; $\Delta f_{i j}$ is the center frequency spacing between connections $i$ and $j ; \gamma$ is the fiber nonlinearity coefficient; and $\beta_{2}$ is the fiber dispersion.

Each link $l$ in the network has two fibers with opposite directions. On each fiber, there are $S$ subcarriers of $C \mathrm{GHz}$ each. There are $M$ modulation format. An all-to-all symmetric bidirectional connection request matrix is given (the extension to the asymmetric case can be obtained by treating each pair's connection as two connections). Based on the bit rate requirement $\Lambda_{i}$ for connection $i$, we calculate $T_{i k}$ as the number of subcarriers required by connection $i$ when assigned modulation format $k$. The same route and subcarriers will be used for the two directions. A route, a modulation format, and a contiguous subcarrier band need to be allocated to each connection to satisfy its bit rate requirement and the corresponding SNR requirement with the selected modulation format. The objective is to minimize the maximum bandwidth, i.e., the maximum subcarrier index on any link assigned to the set of connections. 


\section{Proposed Algorithms and Benchmark}

\subsection{ILP Formulation}

We propose a novel integer linear program (ILP) to minimize the maximum bandwidth used across the network. The ILP takes the following input parameters: $z_{l, n} \in \mathbb{B}=\{0,1\}$, with $z_{l, n}=1$ if node $n$ is an ending node of connection $l ; v_{i, n} \in \mathbb{B}$, with $v_{i, n}=1$ if $n$ is source or destination of connection $i ; \Lambda_{i}$ and $T_{i k}$ as described above; $\mathrm{SNR}_{k}$, the required SNR threshold of modulation format $k ; J_{i, k, h}=\mu \ln \left[\left(0.5 h+T_{i k} / 2\right) /\left(0.5 h-T_{i k} / 2\right)\right]$, the partial nonlinear effect introduced by connection $i$ to another connection when $i$ is assigned modulation format $k$ and the center frequency spacing between them is $h C / 2 ; H_{i, k}=\mu \operatorname{asinh}\left(\rho\left(C T_{i k}\right)^{2}\right)$, the partial nonlinear effect of connection $i$ when it is assigned format $k$; and $\theta$, a large number.

The ILP comprises the following variables: $B_{i} \in \mathbb{N}$, the number of subcarriers allocated to connection $i ; f_{i} \in \mathbb{N}$, the lowest subcarrier index (starting subcarrier index) allocated to connection $i ; F \in \mathbb{N}$, the maximum allocated subcarrier index on any link; $p_{i l} \in \mathbb{B}$, which is 1 if connection $l$ is on the route assigned to connection $i ; q_{\text {in }} \in \mathbb{B}$, which is 1 if node $n$ is on the route assigned to connection $i ; m_{i k} \in \mathbb{B}$, which is 1 if connection $i$ is assigned modulation format $k$; $y_{i j} \in \mathbb{B}$, which is 1 if connections $i$ and $j$ share at least one common link; $u_{i j} \in \mathbb{B}$, which is 1 if $y_{i j}=1$ and $f_{i}+B_{i} \leq f_{j}$; $\Delta_{i j k h} \in \mathbb{B}$, which is 1 if the center frequency spacing between connections $i$ and $j$ is $h C / 2$ and $j$ is assigned modulation format $k$; $w_{i j l} \in \mathbb{R}$, the nonlinear effect to connection $i$ from connection $j$ on link $l$; and $t_{i l} \in \mathbb{R}$, the value of $G_{\mathrm{NLI}}^{l}$ of connection $i$ on link $l$. This notation now allows us to formulate the following optimization problem:

minimize $\xi$

$$
\begin{aligned}
& \text { s.t. } \quad(a) \sum_{k} m_{i k}=1 \\
& { }^{(d)} p_{i l}+p_{j l} \leq 1+y_{i j}, j \neq i \\
& \text { (g) } f_{i}+B_{i}-f_{j} \leq \theta\left(1-u_{i j}\right) \\
& \text { (i) } \sum_{k, h} h \Delta_{i j k h} \leq \theta\left(1-u_{i j}\right)+2\left(f_{j}-1\right)+B_{j}-2\left(f_{i}-1\right)-B_{i} \\
& { }^{(b)} B_{i}=\sum_{k} T_{i k} m_{i k} \quad{ }^{(c)} \sum_{l} p_{i l} z_{l, n}=2 q_{i n}-v_{i, n} \\
& \text { (e) } \xi \geq f_{i}+B_{i}-1 \quad{ }^{(f)} \sum_{h} \Delta_{i j k h}=m_{j k} \quad j \neq i \\
& \text { (h) } f_{j}+B_{j}-f_{i} \leq \theta\left(1-y_{i j}+u_{i j}\right) \quad j \neq i \\
& \text { (j) } \sum_{k, h} h \Delta_{i j k h} \leq \theta\left(1-y_{i j}+u_{i j}\right)+2\left(f_{i}-1\right)+B_{i}-2\left(f_{j}-1\right)-B_{j} \\
& j \neq i \\
& { }^{(k)} w_{i j l} \geq \theta\left(p_{j l}-1\right)+\sum_{k, h} \Delta_{i j k h} J_{j, k, h}, j \neq i \quad{ }^{(l)} t_{i l} \geq \theta\left(p_{i l}-1\right)+\sum_{k} m_{i k} H_{i, k}+\sum_{j} w_{i j l} \quad j \neq i \\
& { }^{(m)} G \sum_{k} m_{i k}\left(1 / \mathrm{SNR}_{k}\right) \geq G_{\mathrm{ASE}}^{0} \sum_{l}\left(p_{i l} N_{l}\right)+N_{l} \sum_{l} t_{i l},
\end{aligned}
$$

where the constraint (a) ensures modulation format allocation; (b) ensures subcarrier allocation; (c) ensures non-loop routing; (d) ensures $y_{i j}=1$ if connections $i$ and $j$ share at least one common link; (e) relates the objective value $\xi$ to the connection bandwidths; (g) and (h) ensure nonoverlapping spectrum allocation for connections $i$ and $j$ if $y_{i j}=1$; (f), (i), and (j) regard the center frequency spacing between connections $i$ and $j$; and (k)-(m) ensure that the SNR requirement of each connection is satisfied.

\subsection{Proposed Heuristics}

The ILP optimizes routing and resource allocation for all connections simultaneously. Because of the many integer constraints, the complexity scales poorly with the number of connections and the size of the networks. To address this, we propose two heuristics: group list (GL) and connection list (CL). In both heuristics, the connections are first sorted by decreasing order of their bit rate requirements $\Lambda_{i}$. In GL, the connections are further grouped in successive nonoverlapping groups of size $\eta \geq 1$. Then, the ILP from Section 3.1 is called successively for each group of $\eta$ connections, accounting for the previously allocated connections. In GL, we include an adaptive SNR margin to protect against interference from $E \geq 1$ future calls, by adding $\sum_{e=i+1}^{i+E} \sum_{k} m_{i k} X_{i e k}$ to constraint (1), in which $X_{i e k}=$ $\mu \ln \left[1+T_{e 1} /\left(T_{i k} / 2\right)\right]$ is the interference from connection $e>i$ to connection $i$ when they use modulation formats $k$ and 1, respectively. If the allocation of the $\eta$ connections is not feasible, $E$ is increased. Through the selection of $\eta$, GL allows trading off complexity and performance. The CL heuristic considers one connection at a time and combines the use of a Dijkstra lowest cost algorithm with an adaptive margin for future calls. The algorithm is summarized in Figure 1.

\subsection{Benchmarks}

The benchmark ILP and heuristics are from [3], which are based on transmission-reach limits. To account for different power levels, the reach with modulation format $k$ is calculated as $\left\lfloor G /\left(\mathrm{SNR}_{k} G_{\mathrm{ASE}}^{0}\right)\right\rfloor$ links, and a guardband of $g$ subcarriers is used to avoid the interference between connections, instead of estimating the actual NLI.

\section{Numerical Results}

We present results for two network topologies [3, Fig. 5]: a small 6-node network (with average link length 455.56 $\mathrm{km}$ ) and the larger 14-node Deutsche Telekom (DT) network. The parameters are set to $\alpha=0.22 \mathrm{~dB} / \mathrm{km}, \gamma=1.32$ 
Initialize: margin window $E=0$; set $p_{i l}=0 \forall i, l$; set ending subcarrier index of connection $i$ as $\phi_{i}=+\infty, \forall i$

(A) for connection $i=1$ to $I$ (sorted order) // Allocate modulation format $k_{i}$, starting subcarrier index $f_{i}$ and the route $r_{i}$ :

for each modulation format $1 \leq k^{\prime} \leq M$, and each starting subcarrier index $1 \leq f^{\prime} \leq S$

for each link $l$, assign $\cos t c_{l}$ :

$c_{l}=\left\{\begin{array}{l}+\infty, \text { if any subcarrier on } l \text { from } f^{\prime} \text { to } f^{\prime}+T_{i k^{\prime}}-1 \text { is unavailable } \\ N_{l}\left(G_{\mathrm{ASE}}^{0}+\mu \operatorname{asinh}\left(\rho T_{i k^{\prime}}^{2}\right)+\mu \sum_{j=1}^{i-1} p_{j l} \ln \frac{\Delta f_{i j}+B_{j} / 2}{\Delta f_{i j}-B_{j} / 2}+\sum_{e=i+1}^{i+E} X_{i e k}\right) \quad \text { otherwise }\end{array}\right.$

end for

if the Dijkstra path $P$ from source to destination of connection $i$ has cost $\sum_{l \in p} c_{l} \leq G / \mathrm{SNR}_{k^{\prime}}$ and $T_{i k^{\prime}}+f^{\prime}-1<\phi_{i}$ then

Update $k_{i} \leftarrow k^{\prime}, f_{i} \leftarrow f^{\prime}, \phi_{i} \leftarrow T_{i k^{\prime}}+f^{\prime}-1$, and $r_{i} \leftarrow P$

end if

end for

Update $p_{i l}=1$ for links $l \in r_{i}$, and set the states of the subcarriers from $f_{i}$ to $\phi_{i}$ on these links as unavailable end for

if any connection does not meet the BER requirement then $E=E+1$; goto (A)

Fig. 1: The CL algorithm, allocating one connection at a time with a margin for $E$ future calls.

$(\mathrm{W} \mathrm{km})^{-1}, \beta_{2}=-21.7 \mathrm{ps}^{2} / \mathrm{km}, F=1.8, v=193 \mathrm{THz}, L=100 \mathrm{~km}$, and $C=6.25 \mathrm{GHz}$. Each connection's BER requirement is $10^{-3}$. We use $M=4$ modulation formats for the subcarriers: BPSK, QPSK, 8-QAM, and 16-QAM. Each connection requires a bit rate that is uniformly distributed from 1 to 30 subcarriers when BPSK is assigned. The resulting bandwidths as a function of the PSD are shown in Fig. 2, averaged over ten connection request matrices.

For the small network, Fig. 2(a) indicates that the benchmark fails for higher PSD, while the ILP provides feasible solution for all PSD values. Furthermore, for the cases where the benchmark is feasible, the ILP leads to up 18\% bandwidth reduction. The CL and GL heuristics are feasible for all PSD values and lead to similar bandwidth utilization. For the large DT network (Fig. 2(b)), the ILP was too complex, so we only consider the CL heuristic, and compare with the benchmark heuristic from [3]. The benchmark heuristics are again only feasible for lower PSD. At higher PSD values, the guardband in the benchmark heuristics is insufficient to account for the NLI, rendering the optimization infeasible. In contrast, CL is feasible for all PSD values and gives the lowest bandwidth utilization, with up to $23 \%$ bandwidth reduction compared to the benchmark.

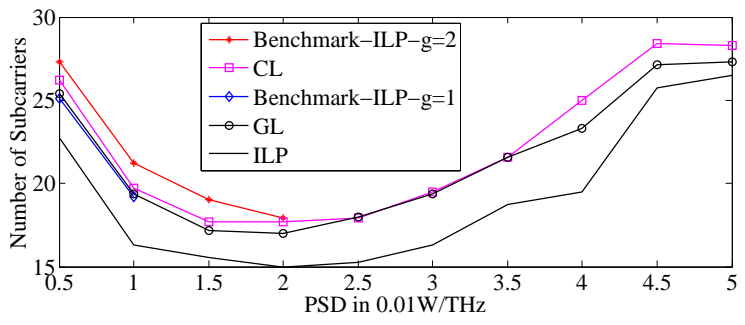

(a) 6-node network results

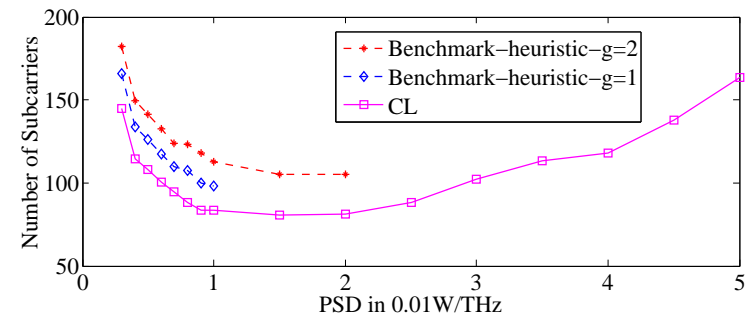

(b) DT network results

Fig. 2: The bandwidth (number of subcarriers) vs. PSD for two networks with the proposed ILP scheme and two heuristics (CL and GL). The benchmark algorithms cannot provide solutions at high PSD, leading to early termination of the corresponding curves.

\section{Conclusions}

By including a realistic NLI model, novel routing and spectrum allocation algorithms were derived for elastic networks based on an ILP optimization formulation. Simulations show that the proposed algorithms guarantee a feasible solution and save up to $23 \%$ in bandwidth compared with state-of-the-art methods, which are based on transmission reach.

\section{References}

1. I.B. Djordjevic and B. Vasic, "Orthogonal Frequency Division Multiplexing for High-speed Optical Transmission," Opt. Expr., vol. 14, pp. 3767-3775 (2006).

2. M. Jinno, B. Kozicki, H. Takara, A. Watanabe, Y. Sone, T. Tanaka, and A. Hirano, "Distance-adaptive Spectrum Resource Allocation in Spectrum-sliced Elastic Optical Path Network," IEEE Commun. Mag., vol. 48, pp. 138-145 (2010).

3. K. Christodoulopoulos, I Tomkos, and E. Varvarigos, "Elastic Bandwidth Allocation in Flexible OFDM-Based Optical Networks," J. Lightw. Technol., vol. 29, pp. 1354-1366 (2011).

4. X. Wan, N. Hua, and X. Zheng, "Dynamic Routing and Spectrum Assignment in Spectrum-Flexible Transparent Optical Networks," J. Opt. Commun. Netw., vol. 4, pp. 603-613 (2012).

5. Z. Zhu, W. Lu, L. Zhang, and N. Ansari,“Dynamic Service Provi- sioning in Elastic Optical Networks With Hybrid Single-/MultiPath Routing," J. Lightw. Technol., vol. 31, pp. 15-22 (2013).

6. D.J. Ives, P. Bayvel, and S.J. Savory,"Physical Layer Transmitter and Routing Optimization to Maximize the Traffic Throughput of a Nonlinear Optical Mesh Network, in Proc. ONDM (2014).

7. H. Beyranvand and J.A. Salehi, "A Quality-of-Transmission Aware Dynamic Routing and Spectrum Assignment Scheme for Future Elastic Optical Networks," J. Lightw. Technol., vol. 31, pp. 3043-3054 (2013).

8. D.J. Ives, A. Lord, P. Wright, and S.J. Savory,"Quantifying the Impact of Non-linear Impairments on Blocking Load in Elastic Optical Networks," in Proc. OFC/NFOEC, W2A.55 (2014).

9. P. Johannisson and E. Agrell, "Modeling of Nonlinear Signal Distortion in Fiber-Optic Networks," J. Lightw. Technol., DOI 10.1109/JLT.2014.2361357 (2014). 